\section{Signaux et contrôles}

Les protéines $G$, grandes et petites, sont les partenaires essentiels de la transmission des signaux à l'intérieur des cellules dont elles règlent pratiquement toutes les fonctions. Les petites protéines $G$ de la famille Rho jouent ainsi un rôle déterminant dans le contrôle de la réonganisation du cytosquelette d'actine. Elles agissent les unes sur les autres, en cascade, et collaborent avec de nombreux partenaires auxquels elles se lient par des domaines protéiques conservés, en un système dont nous avons déjà souligné la complexité. Les formes activées sont ramenées à la quiescence grâce à des cofacteurs stimulant leur activité GTPasique intrinsèque. Contrairement à ce que l'on pensait, la désensibilisation des grandes protéines $G$ trimériques obéit au même mécanisme: elles aussi possèdent leurs activateurs d'activité GTPasique. Les petites protéines $G$ activées semblent véhiculer la kinase Raf à la membrane, conduisant à sa dimérisation par interaction avec un partenaire divalent: c'est cette dimérisation qui pourrait expliquer l'activation de Raf, et donc la poursuite de la transmission du signal, notamment vers la machinerie transcriptionnelle. Cette dernière est contrôlée par d'autres systèmes de kinases, notamment celles dépendantes de l'AMP cyclique dont la synthèse peut être réglée au cours du cycle nycthéméral. Enfin, les hormones stéroïdes, comme les autres ligands de la superfamille des récepteurs mucléaires, sont un moyen plus direct de coupler transcription et signaux extracellulaires... ce qui n'exclut pas un degré insoupçonné de complexité dont témoignent les modes multiples d'action des æestrogènes. Les récepteurs nucléaires jouent aussi un rôle, peut-être essentiel, en couplant le métabolisme intermédiaire et les régulations transcriptionnelles.

\title{
Dissection moléculaire des signalétiques induites par Rac et Cdc42
}

Depuis quelques années, les petites protéines $G$ de la famille Rho, sousgroupe de la vaste famille des protéines Ras, sont le centre d'intérêt de plusieurs équipes de recherche œuvrant dans la biologie cellulaire et moléculaire. Ce n'est pas sans raison car ces petites protéines $G$ occupent une place primordiale dans plusieurs fonctions cellulaires chez les mammifères $\left(m / s n^{\circ} 7, v o l .11, p .1045\right)[1,2]$. Il a été démontré que Rho, Rac et Cdc42 jouent un rôle important dans la réorganisation du cytosquelette d'actine en contrôlant la formation de plaques d'adhérence et de trois structures d'actine impliquées dans les processus de migration et d'adhérence cellulaires. Ainsi, des expériences de micro-injection des formes activées de Cdc42, Rac et Rho dans des fibroblastes de souris ont montré que Cdc42 induit la formation de filopodes ou microspicules, longues et fines extensions de la membrane plasmique constituées de filaments d'actine polymérisée [3, 4]. Rac induit rapidement la polymérisation de l'actine à la membrane plasmique, provoquant le repliement de la membrane sur elle-même (ruffles) [5] et, finalement, Rho entraîne en quelques minutes la formation de fibres de tension (stress fibers) traversant de part et d'autre la cellule [6]. De plus, il existe une hiérarchie entre ces petites protéines $G$ de sorte que Cdc42 conduit à l'activation de Rac qui, à son tour, induit l'activation de Rho.

\section{Contrôle de la prolifération cellulaire}

Les GTPases de la famille Rho affectent non seulement la morphologie de la cellule mais elles semblent éga- lement jouer un rôle important dans le contrôle de la prolifération cellulaire, tout particulièrement dans la phase G1 du cycle cellulaire. De plus, Rac contrôle une voie de transmission du signal essentielle à la transformation cellulaire induite par Ras (figure 1) et participerait également aux mécanismes d'activation du développement métastasique $[7,8]$. Il semble aussi évident que Rho, Rac et Cdc42 contrôlent des voies de transmission du signal impliquées dans le contrôle de la transcription des gènes. En effet, de nombreux groupes ont démontré que Rac et Cdc42 activent la cascade de protéines kinases de la famille JNK/SAPK (c-Jun N-terminal kinase ou stress-activated protein kinase) $\left(\mathrm{m} / \mathrm{s} n^{\circ} 3\right.$, vol. 11, p. 467) ainsi que celle de la famille p38/HOG MAP (mitogene-activated protein) kinase. De même, l'activation du facteur de transcription SRF (serum response factor) est contrôlée à la fois par Rho, Rac et Cdc42 [9]. Les mécanismes biochimiques impliqués dans ces voies de transmission du signal sont toujours aussi peu connus à ce jour. Notamment, il reste à savoir si les signaux conduisant à la réorganisation du cytosquelette d'actine et à la formation des plaques d'adhérence sont différents de ceux impliqués dans le contrôle de la prolifération cellulaire, ou s'ils se recoupent. Ainsi, l'identification des partenaires des petites $G$ dans la transmission de ces signaux a été l'objet de beaucoup de recherches au cours de ces dernières années.

\section{Voies de transmission du signal}

Ce n'est qu'en 1996 que les équipes de S. Narumiya et de K. Kaibuchi (Japon) ont identifié un grand 


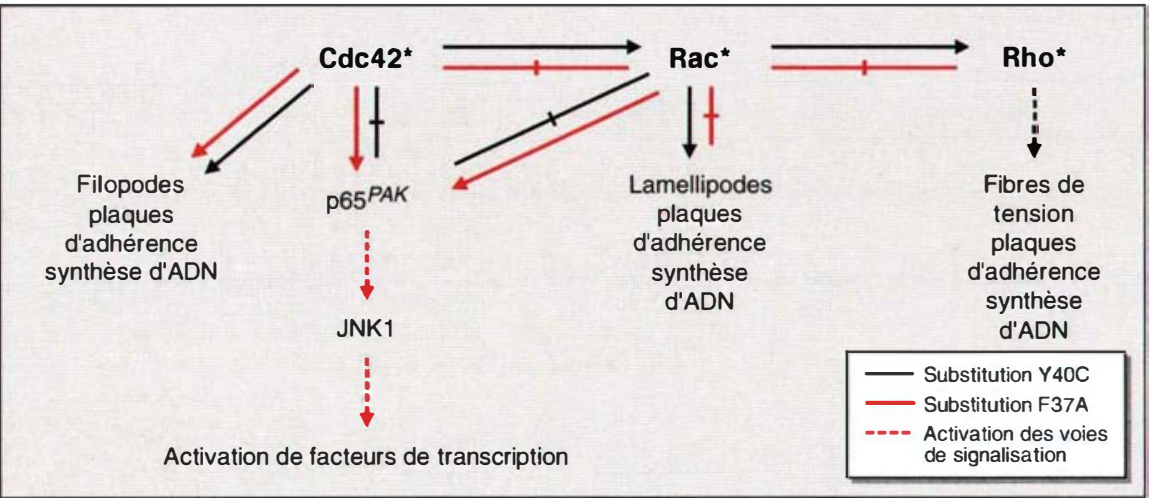

Figure 1. Effets des substitutions Y40C et F37A dans I'activation des voies de transmission du signal relayées par Rac et Cdc42. Lorsque Cdc42L61 et RacL61 (Cdc42* et Rac*), formes constitutivement actives de Cdc42 et de Rac, renferment la substitution $Y 40 C$, elles induisent respectivement les filopodes et lamellipodes, les plaques d'adhérence et la synthèse d'ADN dans des fibroblastes de souris carencés en sérum. De même, ces mutants activent la cascade des GTPases. En revanche, ces mutants Y4OC ne peuvent pas stimuler l'activité kinase de p65 $5^{\text {PAK }}$ et la cascade JNK/SAPK. La substitution F37A dans Cdc42L61 ne bloque que l'activation de la cascade des GTPases tandis que RacL61, renfermant la substitution F37A, ne peut induire ni lamellipodes, ni plaques d'adhérence, ni synthèse d'ADN, ni activer Rho dans les fibroblastes de souris. Cependant, ce mutant peut activer p65 $5^{P A K}$ et la cascade JNK/SAPK. JNK 1: c-jun N-terminal kinase 1. Trait avec pointe de flèche : activation; trait barré : inhibition; ligne pointillée: activation des voies de signalisation.

nombre de cibles potentielles de Rho incluant deux protéines Ser/Thr kinase, la protéine kinase $\mathrm{N}$ et p160 $160 C K$ [2]. Il s'avère que, entre nos mains, pl60 ROCK interagit également in vitro avec la forme activée de Rac, liée au GTP, aussi bien qu'avec celle de Rho [10]. Nous avons aussi montré que Rac contrôle l'activité NADPH oxydase dans les cellules phagocytaires par son interaction directe avec $\mathrm{p} 67^{\text {PHOX }}$, une protéine cytoplasmique appartenant à ce complexe enzymatique $\left(\mathrm{m} / \mathrm{s} n^{\circ} 7\right.$, vol. 11 , p. 1039). De plus, une famille de protéines renfermant la séquence consensus CRIB (Cdc42/Rac interactive binding) a récemment été décrite par notre groupe. Cette famille comprend les protéines Ser/Thr kinase p $65^{P A K}$ et Tyr kinase p $120^{A C K}$ (d'abord identifiées par le groupe de L. Lim, Singapour), de même que le produit du gène du syndrome humain de Wiskott-Aldrich, la protéine WASp $\left(\mathrm{m} / \mathrm{s} n^{\circ} 10\right.$, vol. 12, p. 1173) [11].

Nous avons cherché à définir le rôle de ces cibles potentielles dans les voies de transmission des signaux sant des mutations sélectives dans les régions effectrices de RacL61 et Cdc42L61, des formes activées de ces GTPases (le remplacement en position 61 d'une glutamine par une leucine les rend constitutivement activées par le GTP) [10]. Cette approche, en effet, s'était déjà avérée très fructueuse dans la dissection moléculaire des voies de transmission du signal contrôlé par Ras [12]. Ainsi, nous démontrons clairement que la mutation Y40C dans RacL61 et Cdc42L61 bloque l'interaction de ces GTPases avec les protéines CRIB telles que p65 $5^{P A K}$ et WASp. Cependant, ce remplacement n'affecte pas la liaison de RacL61 avec p67 $7^{\mathrm{PHOX}}$ ou p160 ROCK. En accord avec ces résultats de liaison, les mutants RacL61C40 et Cdc42L61C40 (renfermant la mutation Y40C) sont incapables de stimuler in vivo l'activité Ser/Thr kinase de p $65^{P A K}$ lorsqu'ils sont co-transfectés dans les cellules COS-1. En outre, cette substitution empêche RacL61 et Cdc42L61 d'activer la cascade de kinases de type JNK/SAPK. Ces résultats sont en accord avec ceux des groupes de J. Chant (Cambridge,
MA, USA) et de R. Cerione (Ithaca, NY, USA) qui ont suggéré que l'activation de cette voie des MAP kinases par Rac et Cdc42 serait relayée par p65 ${ }^{P A K}$.

Contrôle de la réorganisation du cytosquelette

Malgré leur incapacité de stimuler l'activité p $65^{P A K}$ et la cascade JNK/SAPK, les mutants Y40C induisent la réorganisation du cytosquelette d'actine (Rac: lamellipodes; Cdc42: filopodes) et la formation de plaques d'adhérence après microinjection dans des fibroblastes de souris. Ainsi, nous mettons en évidence que l'interaction de Rac et Cdc42 avec les protéines CRIB telle que $p 65^{P A K}$ n'est pas requise dans les voies de transmission du signal conduisant à la réorganisation du cytosquelette d'actine et à la formation des plaques d'adhérence. D'un autre côté, la substitution Phe37 $\rightarrow$ Ala (F37A) dans RacL61 et Cdc42L61 n'affecte pas la liaison de ces GTPases avec p65 ${ }^{P A K}$ et, de ce fait, ces mutants induisent l'activité kinase de p $65^{P A K}$ au même niveau que les formes non mutées. Cependant, il apparaît que les mutants F37A n'activent pas aussi bien (environ 2 fois moins) la cascade de kinases de type JNK/SAPK que les GTPases non mutées. Cela suggère donc que p $65^{P A K}$ n'est peut-être pas le seul relais de Rac et Cdc42 dans l'activation de la cascade JNK/SAPK. Le mutant RacL61A37 (renfermant la substitution F37A) est, en outre, incapable d'induire la formation de lamellipodes ou de plaques d'adhérence après micro-injection dans des fibroblastes de souris. La substitution F37A semble donc affecter l'interaction de RacL61 avec un important médiateur encore inconnu, impliqué dans la transmission du signal conduisant à la réorganisation du cytosquelette et des plaques d'adhérence. De manière intéressante, nous avons observé que cette substitution bloque l'interaction de RacL61 avec p160 ${ }^{R O C K}$, suggérant un rôle potentiel de cette kinase dans ces signaux. En revanche, la substitution F37A dans Cdc42L61 ne l'empêche pas d'induire la formation de filopodes ou de 
plaques d'adhérence dans les fibroblastes de souris. Néammoins, elle bloque l'activation de la cascade des GTPases de sorte que Cdc42L61A37 est incapable d'activer Rac et la formation de lamellipodes et RacL61A37 ne peut activer Rho et donc la formation de fibres de tension et de plaques d'adhérence. Finalement, nous avons observé que RacL61C40 et Cdc42L61C40 sont également capables d'induire la synthèse d'ADN dans des fibroblastes de souris. Ces résultats démontrent donc que l'activation de $\mathrm{p} 65^{P A K}$ et de la cascade JNK/SAPK n'est pas indispensable à l'induction par Rac et Cdc42 du passage de la phase $\mathrm{Gl}$ à la phase $\mathrm{S}$ du cycle cellulaire. Alors que la substitution F37A n'affecte pas la capacité de Cdc42 d'induire la synthèse d'ADN, RacL61A37 est, une fois de plus, incapable d'induire cet effet biologique dans les cellules Swiss 3T3. Comme ce mutant est aussi inefficace à stimuler la réorganisation du cytosquelette d'actine et la formation des plaques d'adhérence, nous ne pouvons exclure l'intéressante possibilité que le contrôle de la prolifération cellulaire par Rac résulte de signaux transmis par la formation des plaques d'adhérence ou par la polymérisation de l'actine. Naturellement, cela reste à prouver et une dissection moléculaire plus fine du signal relayé par Rac et Cdc42 sera nécessaire pour répondre à cette question.

\section{Conclusion}

En conclusion, les résultats de cette analyse moléculaire révèlent que le contrôle du cytosquelette d'actine et du cycle cellulaire par Rac et Cdc42 résulte de voies de transmission du signal indépendantes de p $65^{P A K}$ ou de la cascade JNK/SAPK. D'autre part, p $65^{P A K}$ paraît être un effecteur important de Rac et de Cdc42 dans l'activation de JNK/SAPK. Néammoins, il n'est pas impossible que d'autres protéines CRIB dont la fonction biologique est encore inconnue jouent un rôle important dans cette voie de transmission du signal. Ayant éliminé les protéines CRIB comme partenaires de Rac et de Cdc42 dans la régulation du cytosquelette d'actine et du cycle cellulaire, le choix de cibles potentielles se retrouve donc très restreint. La kinase $\mathrm{p} 160^{R(C K}$ se révèle un candidat très intéressant pour jouer un rôle dans la transmission du signal relayé par Rac et le futur promet de nous tenir captivés dans la recherche de nouveaux partenaires de ces petites protéines $G$.

\section{RÉFÉRENCES}

1. Zalcman G, Closson V, Honoré N, Olofsson B, Tavitian A. Participation de la cascade des gènes Rho à la régulation du cytosquelette: rôle possible dans les mécanismes d'oncogenèse. médecine/sciences $1995 ; 11$ : $1551-6$.

2. Reid T, Lang P, Bertoglio J. Vers une compréhension des fonctions effectrices des petites protéines $G$ de la famille Rho et de leur rôle possible dans le système immunitaire. médecine/sciences $1996 ; 12: 1235-9$.

3. Kozma R, Ahmed S, Best A, Lim L. The Ras-related protein Cdc $42 \mathrm{Hs}$ and bradykinin promote formation of peripheral actin microspikes and filopodia in Swiss 3T3 fibroblasts. Mol Cell Biol 1995; 15 : 1942-52.

4. Nobes CD, Hall A. Rho, Rac, and Cdc42 GTPases regulate the assembly of multimolecular focal complexes associated with actin stress fibers, lamellipodia and filopodia. Cell 1995; 81: 53-62.

5. Ridley AI, Paterson HF, Johnston CL, Diekmann D, Hall A. The small GTP-binding protein rac regulates growth factor-induced membrane ruffling. Cell 1992; 70: 401-10.
6. Ridley AJ, Hall A. The small GTP-binding protein rho regulates the assembly of focal adhesions and actin stress fibers in response to growth factors. Cell $1992 ; 70: 389-99$.

7. Qiu RG, Chen J, Kirn D, McCormick F, Symons M. An essential role for Rac in Ras transformation. Nature $1995 ; 374: 457-9$.

8. Michiels F, Habets GG, Stam JC, Van d KR, Collard JG. A role for Rac in Tiam-linduced membrane ruffling and invasion. Nature 1995 ; 375 : 338-40.

9. Vojtek AB, Cooper JA. Rho family members : activators of MAP kinase cascades. Cell $1995 ; 82: 527-9$

10. Lamarche N, Tapon N, Stowers L, Burbelo PD, Aspenström P, Bridges T, Chant J, Hall A. Rac and Cdc42 induce actin polymerization and $\mathrm{Gl}$ cell cycle progression independently of p65PAK and the JNK/SAPK MAP kinase cascade. Cell 1996 $87: 519-29$.

11. Burbelo PD, Drechsel D, Hall A. A conserved binding motif defines numerous candidate target proteins for both Cdc42 and Rac GTPases. I Biol Chem 1995; 270 : 29071-4.

12. White MA, Nicolette $\mathrm{C}$, Minden A, Pol verino A, Van Aelst L, Karin M, Wigler MH Multiple Ras functions can contribute to mammalian cell transformation. Cell 1995 ; $80: 533-41$.

\section{Nathalie Lamarche Alan Hall}

CRC Oncogene and Signal Transduction Group, MRC Laboratory for Molecular Cell Biology and Department of Biochemistry, University College London, Gower Street, London WC1E 6BT, Royaume-Uni.

\section{TIRÉS À PART}

N. Lamarche.

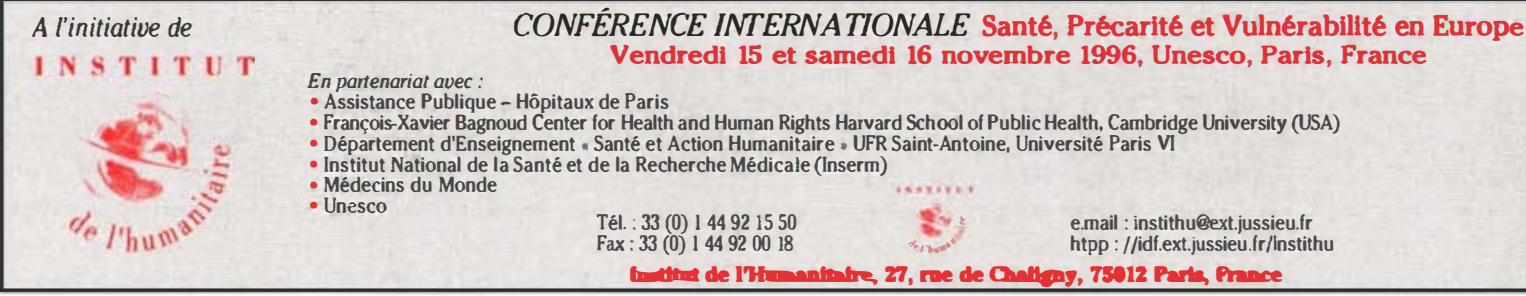

$m / s n^{\circ} 12$, vol. 12 , décembre 96 